\title{
Abordagem bayesiana multivariada para características de crescimento, fertilidade e escores visuais de rebanhos da raça Brangus
}

\author{
Ronyere Olegário de Araújo(1), Cintia Righetti Marcondes ${ }^{(2)}$, Dionéia Magda Everling ${ }^{(3)}$, Tomás Weber(3), \\ Jader Silva Lopes ${ }^{(4)}$, Analía del Valle Garnero(5), Ricardo José Gunski( ${ }^{(5)}$ e Paulo Roberto Nogara Rorato(3)
}

\begin{abstract}
(1)Universidade de Brasília, Faculdade de Agronomia e Medicina Veterinária, Campus Universitário Darcy Ribeiro, Caixa Postal 4.508, CEP 70910-970 Brasília, DF. E-mail: ronyereo@yahoo.com.br ${ }^{(2)}$ Embrapa Pecuária Sudeste, Rodovia Washington Luiz, Km 234, CEP 13560-970 São Carlos, SP. E-mail: cintia@cppse.embrapa.br (3)Universidade Federal de Santa Maria, Centro de Ciências Rurais, Departamento de Zootecnia, Avenida Roraima, no 1.000, CEP 97105-900 Santa Maria, RS. E-mail: dioneiamagda@yahoo.com.br, samotweber@hotmail.com, rorato@smail.ufsm.br ${ }^{(4)}$ Genética e Inovação Agropecuária, Rodovia BR 282, Km 52, CEP 89694-000 Faxinal dos Guedes, SC. E-mail: Jader.Lopes@brasilfoods.com ${ }^{(5)}$ Universidade Federal do Pampa, Campus São Gabriel, Avenida Antônio Trilha, no 1.847, CEP 97300-000 São Gabriel, RS. E-mail: analiagarnero@unipampa.edu.br, ricardogunski@unipampa.edu.br
\end{abstract}

Resumo - O objetivo deste trabalho foi estimar parâmetros genéticos e tendências genéticas e fenotípicas de uma população da raça Brangus. As características peso, circunferência escrotal e escores visuais de conformação, precocidade, musculatura e umbigo, padronizadas para 550 dias de idade, foram avaliadas a partir de 6.789 registros de animais nascidos de 288 touros e 5.949 vacas, entre 1991 e 2001, em 49 fazendas localizadas nas regiões Centro-Oeste, Sudeste e Sul do Brasil. Para a estimação dos parâmetros, das correlações e das tendências genéticas, foi adotado o modelo animal linear-limiar hexacaracterística. As estimativas de herdabilidade direta foram de 0,39 e 0,27 , para peso e circunferência escrotal, respectivamente, e de $0,22,0,20$, 0,23 e 0,33 para conformação, precocidade, musculatura e umbigo, o que indica considerável variação genética aditiva e que é possível obter ganho genético por meio da seleção. As correlações genéticas entre peso e circunferência escrotal com os escores de conformação, precocidade e musculatura mostram a possibilidade de resposta correlacionada. As tendências genéticas estimadas indicam grande contribuição de fontes de variação não genéticas para todas as características no período estudado, e apontam a necessidade de melhoria das condições ambientais, para que os animais expressem todo seu potencial genético.

Termos para indexação: amostragem de Gibbs, características morfológicas, modelo de limiar, precocidade de acabamento.

\section{Bayesian multivariate approach for growth, fertility and visual score traits of Brangus cattle herds}

\begin{abstract}
The objective of this work was to estimate genetic parameters, and genetic and phenotypic trends of a Brangus breed population. Weight, scrotal circumference, and visual scores of conformation, precocity, musculature, and navel, adjusted to 550 days of age, were evaluated from records of 6,789 animals sired by 288 bulls and 5,949 dams, born between 1991 and 2001 in 49 farms located in the Center-West, Southeast, and South regions of Brazil. To estimate parameters, correlations, and genetic trends, a six-trait, linear-threshold animal model was adopted. Direct heritability estimates were 0.39 and 0.27 for weight and scrotal circumference, respectively, and 0.22, 0.20, 0.23, and 0.33 for conformation, precocity, musculature, and navel, indicating considerable additive genetic variation, and that it is possible to obtain genetic gain by selection. Genetic correlations between weight and scrotal circumference with the scores of conformation, precocity, and musculature show the possibility of correlated responses. Genetic trends indicate great contribution of non-genetic sources of variation in the evaluated traits, which leads to the necessity of improving environmental conditions, in order for the animals to fully express their genetic potential.
\end{abstract}

Index terms: Gibbs sampling, morphological traits, threshold model, early finishing.

\section{Introdução}

A raça Brangus é utilizada no Brasil para a produção de carne e em cruzamentos com animais adaptados, o que torna importante a identificação e disponibilização, para o mercado, de animais precoces, geneticamente superiores (Oliveira et al., 2011). A seleção para 
precocidade de acabamento está em evidência, em estudos com bovinos de corte, pois o encurtamento do ciclo de produção, principalmente em sistemas a pasto, é um grande passo para aperfeiçoar os índices produtivos da bovinocultura de corte brasileira. Neste contexto, escores visuais poderiam ser utilizados para a seleção de tipos morfológicos que atendam às exigências do mercado (Faria et al., 2009), ao se considerar a variabilidade genética em rebanhos puros (Boligon et al., 2009) e mestiços (Araújo et al., 2010).

Estas avaliações consistem em classificar os animais pela atribuição de escores de conformação (C), precocidade $(\mathrm{P})$ e musculatura $(\mathrm{M})$, com a vantagem de baixo custo (Koury Filho et al., 2003). Outra característica morfológica importante, sobretudo para as raças com grau de sangue zebuíno, é o tamanho do umbigo (U), avaliado por meio de escores à desmama e ao sobreano (Koury Filho et al., 2003). A característica tamanho do umbigo, segundo Araújo et al. (2010), apresenta considerável variação genética, o que indica ser possível obter ganho genético por meio da seleção.

As características reprodutivas são fundamentais para o melhoramento genético de bovinos de corte, pois podem limitar a intensidade de seleção e interferir no intervalo entre gerações, além de serem economicamente relacionadas ao sistema de produção. Entre as características indicadoras de fertilidade e precocidade sexual, a única amplamente empregada nos programas de melhoramento, em razão da facilidade de mensuração e da variância genética aditiva (Cyrillo et al., 2001), é a circunferência escrotal (Boligon et al., 2009). Entretanto, estudos que relacionam escores visuais com o desempenho reprodutivo de zebuínos, ou compostos de zebuínos como a raça Brangus, são escassos na literatura (Faria et al., 2009).

O objetivo deste trabalho foi estimar parâmetros genéticos, tendências genéticas e fenotípicas para as características peso, circunferência escrotal e escores visuais de conformação, precocidade, musculatura e umbigo, padronizadas para 550 dias de idade, em uma população da raça Brangus.

\section{Material e Métodos}

Foram analisadas as características peso (P550), circunferência escrotal (CE) e escores visuais (EVs) de conformação $(\mathrm{C})$, precocidade $(\mathrm{P})$, musculatura $(\mathrm{M}) \mathrm{e}$ umbigo (U), padronizadas para 550 dias de idade, de uma população multirracial da raça Brangus, composta por animais com idade média de 517,45 $\pm 68,4$ dias, nascidos entre 1991 e 2001, em 49 fazendas localizadas nas regiões Centro-Oeste, Sudeste e Sul do Brasil. O escore $\mathrm{C}$ prediz o quanto o animal produziria de carne se fosse abatido naquele momento; o $\mathrm{P}$, o potencial de o animal chegar a um grau de acabamento mínimo de carcaça, com peso vivo não elevado, a partir da avaliação do acúmulo de gordura no costado, da inserção de cauda e da ponta da maçã no peito; o escore $\mathrm{M}$ informa quanto à convexidade e à distribuição das massas musculares em pontos chaves do animal, como quartos traseiros, lombo, paleta e antebraço; e o U, quanto à forma do umbigo, avaliada a partir de uma referência de seu tamanho e posicionamento, características de grande importância, especialmente no Brasil, em que a maioria dos animais é criada em regime de pasto.

As pontuações nas avaliações dos EVs variaram de 1 a 5. Cada animal foi avaliado em relação ao seu grupo de manejo, tendo-se atribuído o escore 3 a animais intermediários, que servem como base de comparação para os demais dentro do grupo. Para o escore de umbigo, atribuiu-se o escore 1 ao animal com umbigo muito penduloso (distante da região ventral), e 5 ao animal com umbigo curto e bem direcionado (Koury Filho et al., 2003).

Para análise da consistência dos dados (SAS Institute, 2003), foram criadas as variáveis: estação de nascimento, tendo-se considerado animais nascidos de janeiro a março como 1, de abril a junho como 2, de julho a setembro como 3 e de outubro a dezembro como 4; grupos de contemporâneos (GCs), que reuniu animais de mesma estação de nascimento, mesma fazenda e mesmo grupo de manejo ao sobreano; grupo genético da vaca (GGV) e grupo genético do touro (GGT), ambos em classes formadas pela contribuição da fração Angus (A) e Nelore (N), o que totalizou quatro grupos diferentes (Tabela 1).

Tabela 1. Relação entre os grupos genéticos, tendo-se considerado a contribuição das raças Angus (A) e Nelore (N), com os respectivos números de observações para a população estudada.

\begin{tabular}{lcccc}
\hline Grupo & Número de & \multicolumn{3}{c}{ Composição racial } \\
\cline { 3 - 5 } genético & animais $^{(1)}$ & Vaca & Touro & Animal \\
\hline 1 & 1.024 & $1 \mathrm{~A} \times 0 \mathrm{~N}$ & $1 / 4 \mathrm{~A} \times 3 / 4 \mathrm{~N}$ & $5 / 8 \mathrm{~A} \times 3 / 8 \mathrm{~N}$ \\
2 & 3.017 & $5 / 8 \mathrm{~A} \times 3 / 8 \mathrm{~N}$ & $5 / 8 \mathrm{~A} \times 3 / 8 \mathrm{~N}$ & $5 / 8 \mathrm{~A} \times 3 / 8 \mathrm{~N}$ \\
3 & 712 & $1 / 2 \mathrm{~A} \times 1 / 2 \mathrm{~N}$ & $3 / 4 \mathrm{~A} \times 1 / 4 \mathrm{~N}$ & $5 / 8 \mathrm{~A} \times 3 / 8 \mathrm{~N}$ \\
4 & 2.036 & $1 / 4 \mathrm{~A} \times 3 / 4 \mathrm{~N}$ & $1 \mathrm{~A} \times 0 \mathrm{~N}$ & $5 / 8 \mathrm{~A} \times 3 / 8 \mathrm{~N}$ \\
\hline
\end{tabular}

(1)Número de animais com registros no arquivo final. 
Foram eliminados do arquivo os machos castrados, as fêmeas, os grupos de contemporâneos com menos de cinco observações e os touros com menos de cinco filhos. Também foram descartados animais fora do limite de $\pm 2,5$ desvios-padrão, em relação à média de P550 na população. A análise de variância foi efetuada com uso do procedimento "stepwise", para escolha do melhor modelo fixo para cada uma das características, com base no valor máximo de R-quadrático a partir da concatenação dos efeitos fixos. Este procedimento permite a inclusão ou a exclusão de efeitos no modelo de análise, mudanças na ordem de entrada dos efeitos no modelo, e a verificação de possíveis confusões e interações entre efeitos.

Tanto a heterozigose direta (HD) quanto a materna (HM) foram calculadas como: $\mathrm{h}_{\mathrm{ij}}=\alpha^{\mathrm{ti}} \alpha^{\mathrm{vj}}+\alpha^{\mathrm{tj}} \alpha^{\mathrm{vi}}$, em que $\alpha^{\text {ti }}$ e $\alpha^{\text {vi }}$, e representaram a proporção do gene da raça "i" no pai e na mãe do animal, respectivamente.

Após a edição, o arquivo ficou constituído de 6.789 registros de animais, os quais foram agrupados em 583 grupos de contemporâneos. A matriz de parentesco constituiu-se de 10.467 animais.

Para a estimação dos parâmetros, das correlações e das tendências genéticas, foram utilizados programas sob enfoque bayesiano (Misztal et al., 2008). No arquivo de parâmetros, os "priors", que determinam os elementos das matrizes referentes aos efeitos residuais e aditivos para as características, foram obtidos por meio de análises frequentistas hexacaracterísticas. $\mathrm{Na}$ análise, foi adotado modelo animal hexacaracterística, que teve como aleatórios os efeitos genéticos aditivos diretos e residuais; como fixos, os efeitos do ano de nascimento, dos GCs, do GGV, do GGT, da HD e da HM; e como covariáveis, a idade ao sobreano e a idade da vaca ao parto.

Em termos matriciais, as equações de modelos mistos para obtenção da melhor predição linear não viesada (BLUP) podem ser descritas como: $\mathrm{y}=\mathrm{X} \beta+\mathrm{Za}+\varepsilon$, em que y é o vetor das observações de todas as características (P550, CE, C, P, M e U); $\mathrm{X}$ é a matriz de incidência dos efeitos fixos (ano, GCs, GGV, GGT, HD e HM); $\beta$ é o vetor dos efeitos fixos; $Z$ é a matriz de incidência do efeito genético direto de cada animal; a é o vetor de efeitos genéticos diretos aleatórios; e $\varepsilon$ é o vetor de efeitos residuais aleatórios. As pressuposições assumidas do modelo foram: $\mathrm{E}[\mathrm{y}]=\mathrm{Xb} ; \operatorname{Var}(\mathrm{a})=\mathrm{A} \otimes \sum_{\mathrm{a}} ; \operatorname{Var}(\varepsilon)=\mathrm{I}_{\mathrm{N}} \otimes \sum_{\varepsilon}, \mathrm{em}$ que $\sum_{\mathrm{a}}$ é a matriz de covariância genética aditiva direta entre as características; $\sum_{\varepsilon}$ é a matriz de covariâncias residuais; A é a matriz de parentesco; I é a matriz identidade; $\mathrm{N}$ é o número de animais com registros; e $\otimes$ é o produto Kronecker entre matrizes.

Em seguida, o arquivo de parâmetros foi submetido a programa que utiliza metodologia bayesiana para características contínuas e categóricas (Misztal et al., 2008), tendo-se seguido o mesmo modelo matricial adotado na análise frequentista. Para a análise bayesiana, foi implementada uma cadeia de 1 milhão de rodadas com descarte inicial de 20 mil amostras. As amostragens (a cada 100 ciclos) e as análises post-Gibbs foram realizadas no programa PostgibbsF90 (Tsuruta \& Misztal, 2006). A convergência da cadeia de Markov foi verificada por meio dos critérios de Heidelberger \& Welch e de Geweke, para as colunas com os valores das variâncias de todas as características. Esses critérios forneceram valores entre 0,002 e 0,006 (Passed) e entre -1,21 e 1,54 (valor de Z), respectivamente. Estes valores confirmam a convergência da cadeia de Markov, o que já era esperado, uma vez que esta análise seguiu o critério de Raftery \& Lewis quanto à referida cadeia. Estes critérios foram estimados por meio do pacote estatístico Bayesian output analysis (BOA) do programa R, versão 2.10.1 (Smith, 2007).

Em relação ao aspecto limiar do modelo, considerouse que a escala subjacente apresentava distribuição normal contínua, representada por: $\mathrm{U} \mid \theta \sim \mathrm{N}(\mathrm{W} \theta$, $\left.I \sigma_{\mathrm{e}}^{2}\right)$, em que $\mathrm{U}$ é o vetor da escala base de ordem r; $\theta=$ ( $\beta$ 'e a') é o vetor dos parâmetros de locação de ordem $\mathrm{s}, \operatorname{com} \beta$ definido sob o ponto de vista frequentista como efeitos fixos e ordem s, em que: a representa os efeitos aleatórios genéticos aditivos diretos; $\mathrm{W}$ é a matriz de incidência conhecida, de ordem r por s; I é a matriz identidade de ordem r por r; e $\sigma_{\mathrm{e}}^{2}$ é a variância residual. Quando se considera que a variável na distribuição subjacente não é observável, a parametrização $\sigma_{\mathrm{e}}^{2}=1$ é geralmente adotada para que se possa identificá-la na função de verossimilhança.

Segundo Boligon et al. (2009), as características categóricas são determinadas por variáveis contínuas não observáveis, em escala subjacente, em que são fixados valores iniciais de limiares, tal que: $\mathrm{t} 1<\mathrm{t} 2 \mathrm{~K}<\mathrm{tj}$ - 1 , com t0 $=-\infty$ e tj $=\infty$, em que $\mathrm{j}$ é o número de categorias. Os dados observáveis são dependentes da variável subjacente, que é limitada entre dois limiares não observáveis (Gianola \& Foulley, 1983). Assim, as categorias ou os escores de yi (características 
categóricas), para cada animal i, são definidos por Ui, na escala subjacente: $\mathrm{y} 1=(1) \mathrm{t} 0<\mathrm{Ui} \leq \mathrm{t} 1 ;(2) \mathrm{t} 1<\mathrm{Ui} \leq \mathrm{t} 2$; (3) $\mathrm{t} 2<\mathrm{Ui} \leq \mathrm{t} 3$; (4) $\mathrm{t} 3<\mathrm{Ui} \leq \mathrm{t} 4$; (5) $\mathrm{t} 4<\mathrm{Ui} \leq \mathrm{t} 5$, para $\mathrm{i}=1$, $\ldots, n$, em que $n$ é o número de observações. Após as especificações dos limiares t 0 a t5, é necessário que um dos limiares (de t1 a t4) seja ajustado a uma constante arbitrária. No presente trabalho, como em Boligon et al. (2009), considerou-se $t 1=0$, e o vetor de limiares estimáveis foi definido como $\mathrm{t}=\mathrm{t} 2$; $\mathrm{t} 3$ e $\mathrm{t} 4$.

As saídas foram trabalhadas no SAS, para calcular as estimativas de herdabilidade $\left(\mathrm{h}_{\mathrm{a}}^{2}\right)$ e as correlações genéticas $\left(\mathrm{r}_{\mathrm{a}}\right)$, bem como para obter a região de credibilidade a $95 \%$ para $h_{a}^{2}$ e $r_{a}$. O arquivo gerado pelo SAS foi submetido a análises, a posteriori, no programa Gibanal (Van Kaam, 1997), para estimar a correlação serial entre as amostras e obter as densidades marginais posteriores, para edição dos gráficos de distribuição posterior das $h_{a}^{2}$ de cada característica e das $r_{a}$ de todas as associações.

Para a determinação da tendência genética das características avaliadas, foram utilizados os valores correspondentes às médias aritméticas dos valores genéticos de cada grupo de animais, dentro de seu respectivo ano de nascimento, ponderadas pelo número de observações, obtidas pelo procedimento Proc Reg (SAS Institute, 2003), com uso da seguinte equação: $\mathrm{Y}_{\mathrm{i}}=\mathrm{b}_{\mathrm{o}}+\mathrm{b}_{1} \mathrm{x}_{\mathrm{i}}+\varepsilon_{\mathrm{i}}$, em que $\mathrm{Y}_{\mathrm{i}}$ é o valor genético para as características avaliadas do iésimo ano de nascimento; $\mathrm{b}_{\mathrm{o}}$ é o intercepto; $\mathrm{b}_{1}$ é o coeficiente angular da reta; $\mathrm{x}_{\mathrm{i}}$ é o iésimo ano de nascimento; e $\varepsilon_{\mathrm{i}}$ é o erro aleatório.

\section{Resultados e Discussão}

Os valores pontuais de média, moda e mediana (Me, Mo e Med), para as herdabilidades diretas $\left(\mathrm{h}^{2}{ }_{\mathrm{a}}\right)$, apresentaram pequena variação em comparação aos valores mínimos e máximos (Tabela 2). As magnitudes das $\mathrm{h}_{\mathrm{a}}^{2}$ obtidas para P550 estão em consonância com os valores $(0,35$ e 0,42$)$ observados por Toral et al. (2007) e por Madureira et al. (2009), respectivamente, os quais utilizaram a mesma metodologia adotada no presente trabalho, para rebanhos da raça Nelore.

No entanto, o resultado para $\mathrm{CE}$ foi inferior ao 0,42 relatado por Yokoo et al. (2007), que também implementaram metodologia bayesiana, em estudo da $\mathrm{CE}$, para rebanhos Nelore. Assim, as estimativas de $\mathrm{h}_{\mathrm{a}}^{2}$ obtidas para P550 e CE indicam grande participação da variação genética aditiva na transmissão dessas características, e que o cruzamento e a seleção podem ser utilizados como ferramenta para modificar a composição genética dos rebanhos e promover melhorias, tanto produtivas quanto reprodutivas, no progresso genético, no decorrer dos anos.

As estimativas de $\mathrm{h}_{\mathrm{a}}^{2}$ encontradas para $\mathrm{C}, \mathrm{P}$ e M foram próximas, tendo variado entre 0,20 e 0,23 (Tabela 2). Esse resultado é indicativo, em um primeiro momento, da existência de variabilidade genética moderada na população, com possibilidade de se obter ganho genético por meio da seleção, para estas características. Porém, cabe destacar que não existe padronização nos sistemas de avaliação desses escores, nos diversos sistemas de criação, o que limita a sua recomendação como bons marcadores fenotípicos para uso na seleção, visto que, até em raça pura, como a Nelore, a variação constatada é grande. As estimativas de $\mathrm{h}_{\mathrm{a}}^{2}$ obtidas para os escores foram superiores às relatadas por Forni et al. (2007), em trabalho com animais da raça Nelore, que obtiveram valores de $0,12,0,15$ e 0,12 para $\mathrm{C}, \mathrm{P}$ e $\mathrm{M}$, respectivamente, Weber et al. (2009), que avaliaram animais da raça Aberdeen Angus, encontraram valores de $0,13,0,11$ e 0,16 para C, P e M. Costa et al. (2008) e Queiroz et al. (2011), ambos em estudos com animais da raça Brangus, à desmama, obtiveram valores similares para $\mathrm{C}, \mathrm{P}$ e $\mathrm{M}$, de 0,17, 0,16 e 0,15,

Tabela 2. Correlação serial, análises descritivas para variância genética aditiva e estimativas de herdabilidade obtidas para as características após análises com o programa Gibanal.

\begin{tabular}{|c|c|c|c|c|c|c|c|}
\hline \multirow[t]{2}{*}{ Característica } & \multirow[t]{2}{*}{ Correlação serial } & \multicolumn{3}{|c|}{ Variância genética } & \multicolumn{3}{|c|}{ Herdabilidade } \\
\hline & & $\mathrm{Me} \pm \mathrm{EMC}$ & Moda & Mediana & $\mathrm{Me} \pm \mathrm{EMC}$ & Moda & Mediana \\
\hline Peso aos 550 dias $(\mathrm{kg})$ & $-0,033$ & $338,4 \pm 3,894$ & 328,1 & 333,8 & $0,392 \pm 0,0006$ & 0,381 & 0,289 \\
\hline Circunferência escrotal $(\mathrm{cm})$ & 0,013 & $1,999 \pm 0,014$ & 1,975 & 1,972 & $0,276 \pm 0,0004$ & 0,270 & 0,273 \\
\hline Escore de conformação & $-0,048$ & $0,155 \pm 0,001$ & 0,154 & 0,153 & $0,215 \pm 0,0004$ & 0,210 & 0,212 \\
\hline Escore de precocidade & $-0,093$ & $0,094 \pm 0,0001$ & 0,093 & 0,093 & $0,202 \pm 0,0002$ & 0,210 & 0,204 \\
\hline Escore de musculatura & 0,081 & $0,169 \pm 0,001$ & 0,155 & 0,166 & $0,232 \pm 0,0005$ & 0,223 & 0,230 \\
\hline Escore de umbigo & 0,068 & $0,243 \pm 0,001$ & 0,234 & 0,239 & $0,327 \pm 0,0006$ & 0,328 & 0,324 \\
\hline
\end{tabular}

$\mathrm{Me} \pm \mathrm{EMC}$, médiaterro de Monte Carlo. 
respectivamente. Contudo, os resultados apresentados no presente trabalho foram inferiores aos observados por Jorge Júnior et al. (2004), de 0,33 para C, 0,39 para $\mathrm{P}$ e 0,34 para $\mathrm{M}$, em pesquisa com animais Nelore.

Observou-se variação genética para o escore de umbigo, com valor de $h_{a}^{2}(0,32)$ superior aos relatados na literatura e semelhante ao 0,29 obtido por Koury Filho et al. (2003), ao avaliar a raça Nelore, e ao 0,24 estimado por Viu et al. (2002), para animais da raça Brangus. A discussão sobre os EVs refere-se, em grande parte, a animais de raças puras, com uso de metodologia frequentista (REML), por não se ter conhecimento de trabalhos publicados que abordassem essas características em rebanhos da raça Brangus e sob enfoque bayesiano.

De certa forma, análises multicaracterísticas são mais apropriadas para avaliações de características pós-desmama, pois removem o viés causado pelo efeito da seleção que geralmente ocorre neste período. Assim, as estimativas de herdabilidade de média magnitude, encontradas no presente trabalho, indicam que parte considerável da variação existente entre os animais, para estas características, esteve sob a influência de componente genético aditivo e que a população responderia de maneira significativa à seleção direta, ao se utilizar P550, CE e EVs como critérios. Ressaltase que os valores estimados para $U$, pela sua magnitude, responderiam à seleção para redução do tamanho do umbigo, o que funcionalmente tem grande importância, principalmente para animais com maior predominância de constituição zebuína (Araújo et al., 2010).

As Me, Mo e Med foram muito próximas entre si para todas as associações genéticas $\left(r_{a}\right)$ entre as características, o que também indica simetria da distribuição posterior (Tabela 3). A $r_{a}$ estimada entre as características P550 e CE apresentou valor alto e positivo. As $r_{a}$ médias a posteriori, entre a CE e os EVs, foram positivas e de moderada magnitude.

Apesar de a seleção direta para CE ser a forma mais eficiente de se obter melhorias nesta característica, observou-se que a seleção para os EVs ao sobreano, principalmente quanto à $\mathrm{C}$, pode ser boa alternativa para melhorar a fertilidade e a precocidade sexual dos rebanhos, além de permitir a seleção de animais com biótipos compatíveis com o sistema de produção. Altas $r_{\mathrm{a}}$ também foram observadas entre C, P e M, com amplitude de 0,60 a 0,85 (Tabela 3 ), o que está de acordo com a variação $(0,92$ a 0,95$)$ relatada por Costa et al. (2008), para animais da raça Brangus, e com os valores $(0,71$ a 0,95$)$ obtidos por Koury Filho et al. (2010) em animais da raça Nelore. Como esses escores descrevem o potencial para a produção de carne do animal, como um todo, ou seja, são componentes diretos do peso corporal do animal, aquele que se mostrar de mais fácil mensuração, com maior herdabilidade e menor influência do avaliador, deve ser recomendado como critério de seleção. Contudo, a $r_{a}$ do $U$ com as demais características foi baixa, tendo variado de 0,02 a 0,23 ,

Tabela 3. Correlação serial, análises descritivas e região de credibilidade a 95\%, para todas as associações entre as características avaliadas após análise com o programa Gibanal.

\begin{tabular}{|c|c|c|c|c|c|}
\hline Associação(1) $^{(1)}$ & Correlação serial & Mediana & Moda & Mediana & Credibilidade a $95 \%(2)$ \\
\hline P550 x CE & 0,167 & 0,747 & 0,764 & 0,753 & 0,59 a 0,86 \\
\hline P550 x C & $-0,037$ & 0,618 & 0,640 & 0,626 & 0,40 a 0,79 \\
\hline $\mathrm{P} 550 \times \mathrm{P}$ & 0,023 & $-0,098$ & $-0,121$ & $-0,100$ & $-0,36$ a 0,17 \\
\hline P550 x M & $-0,017$ & 0,172 & 0,205 & 0,174 & $-0,09$ a 0,42 \\
\hline P550 x U & 0,005 & 0,081 & 0,085 & 0,084 & $-0,18$ a 0,32 \\
\hline $\mathrm{CE} \times \mathrm{C}$ & 0,072 & 0,564 & 0,601 & 0,570 & 0,28 a 0,79 \\
\hline CE $x$ P & 0,018 & 0,315 & 0,340 & 0,323 & 0,03 a 0,55 \\
\hline CE $\times M$ & 0,028 & 0,373 & 0,391 & 0,381 & 0,10 a 0,60 \\
\hline CE X U & 0,118 & 0,226 & 0,251 & 0,229 & $-0,04$ a 0,48 \\
\hline $\mathrm{C} \times \mathrm{P}$ & 0,037 & 0,605 & 0,626 & 0,617 & 0,38 a 0,78 \\
\hline $\mathrm{C} \times \mathrm{M}$ & 0,029 & 0,693 & 0,743 & 0,703 & 0,48 a 0,84 \\
\hline $\mathrm{C} \times \mathrm{U}$ & 0,014 & 0,084 & 0,133 & 0,089 & $-0,22$ a 0,37 \\
\hline $\mathrm{P} \times \mathrm{M}$ & 0,103 & 0,850 & 0,883 & 0,857 & $-0,73$ a 0,93 \\
\hline $\mathrm{P} \times \mathrm{U}$ & $-0,009$ & 0,015 & 0,013 & 0,012 & $-0,26$ a 0,31 \\
\hline$M \times U$ & 0,024 & 0,129 & 0,142 & 0,131 & $-0,16$ a 0,40 \\
\hline
\end{tabular}

(1)P550, peso (kg) ajustado para 550 dias de idade; $\mathrm{CE}$, circunferência escrotal $(\mathrm{cm})$ ajustada para 550 dias de idade; $\mathrm{C}$, escore de conformação; P, escore de precocidade; M, escore de musculatura; U, escore de umbigo. ${ }^{(2)}$ Limite inferior igual a 2,5\% e limite superior igual a $97,5 \%$. 
semelhantemente à variação de 0,09 a 0,16 reportada por Araújo et al. (2010), para rebanhos multirraciais Angus-Nelore. Esse comportamento indica, como evidenciado por estes últimos autores, que a seleção para essas características, não levaria, necessariamente, a um maior escore para umbigo.

Observou-se que, de modo geral, as médias dos valores genéticos diretos (VGD) e as médias fenotípicas (MF) de P550, CE e EVs, estratificados por ano avaliado, variaram entre os anos (Figura 1). Este comportamento oscilatório do VGD médio anual para a característica P550, com diferença superior a $5 \mathrm{~kg}$ entre o VGD mais positivo (1995) e o mais negativo (1998), pode ser atribuído à diversidade genética do pedigree da população estudada, isto é, ser apenas efeito da diferença de composição racial entre as gerações, o que não permite afirmar, em um primeiro momento, que não estaria havendo um programa direcionado para a melhoria desta característica.

A tendência genética direta para P550 (Figura 1) foi de 81 gramas por ano, valor que representa $0,08 \%$

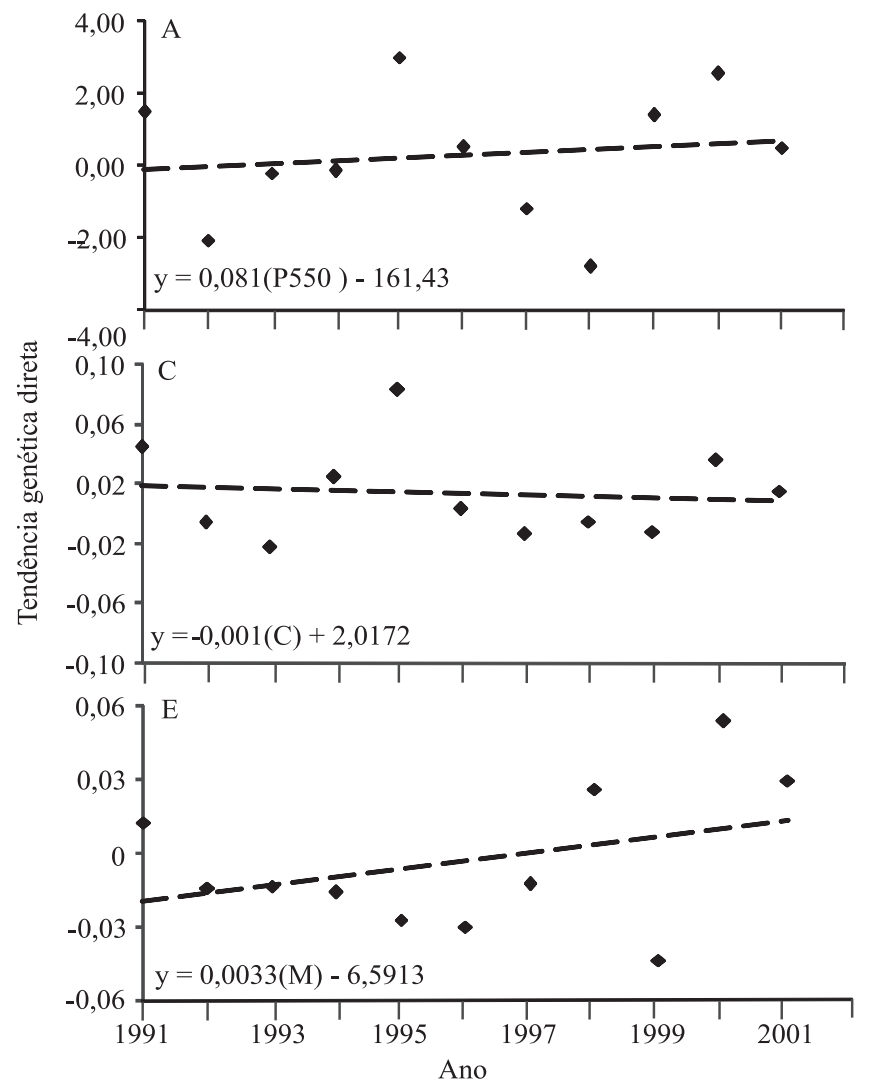

nas médias anuais de P550, em termos de mudança genética. Este resultado foi inferior aos 219 gramas por ano obtidos por Laureano et al. (2011), para rebanhos da raça Nelore, e aos 345 gramas por ano relatados por Mucari \& Oliveira (2003), para rebanhos Tabapuã. Entretanto, Ferraz Filho et al. (2002), ao trabalhar com rebanhos da raça Nelore Mocho criados nas regiões Nordeste, Centro-Oeste e Sudeste do Brasil, reportaram valores superiores, em que os ganhos anuais em decorrência da combinação dos fatores genéticos representaram $1,09 \%$, da média fenotípica para P550 nessas idades.

Para CE, a tendência genética direta (TGD) (Figura 1) foi de $0,027 \mathrm{~cm}$ por ano, o que representa mudança média anual de $0,06 \%$, superior à observada por Laureano et al. (2011) ao avaliar a CE, no período de 1984 a 1995, em rebanhos da raça Nelore. Estes autores relataram TGD para CE igual a 0,011 , o que representa $0,04 \%$ da mudança média anual. No entanto, Cyrillo et al. (2001) utilizaram informações de bovinos da raça Nelore e obtiveram valor semelhante de mudança

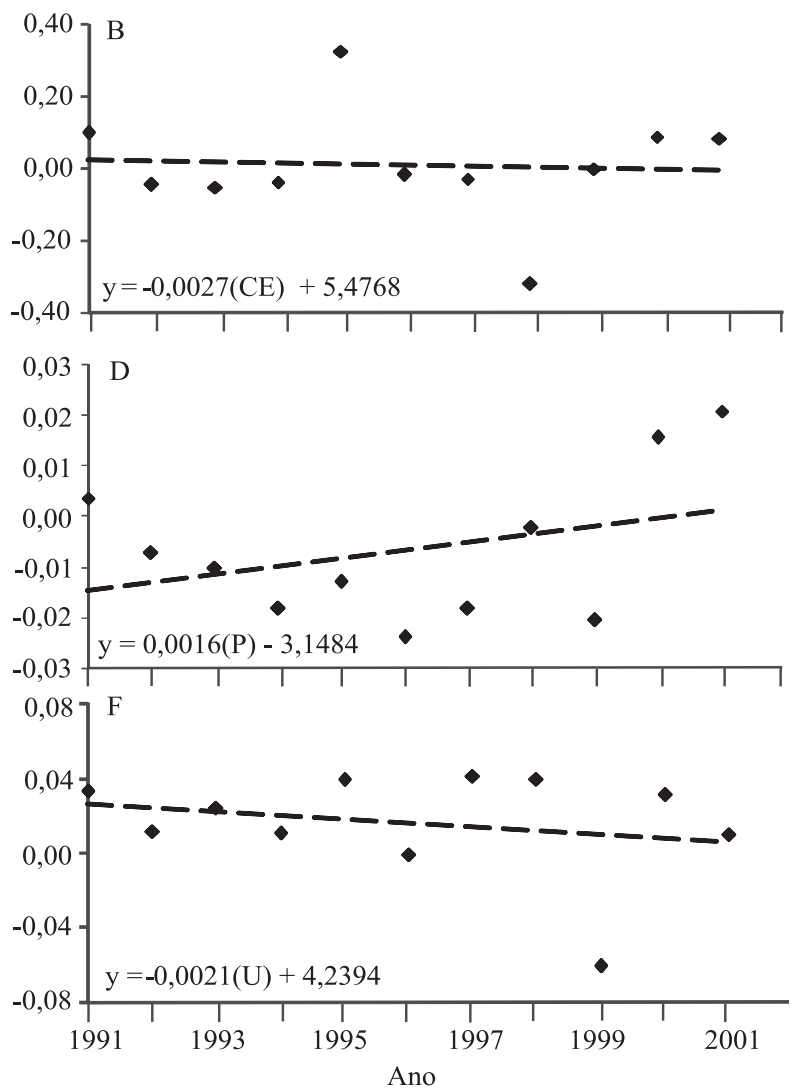

Figura 1. Tendência genética direta para: A, peso (P550, kg); B, circunferência escrotal $(\mathrm{CE}, \mathrm{cm})$; C, conformação (C); D, precocidade $(\mathrm{P})$; E, musculatura (M); e F, umbigo (U), estratificados por unidade de escore, aos 550 dias de idade. 
genética para CE $(0,31 \mathrm{~cm}$ por ano). Segundo Smith (1983), é possível obter taxa de mudança genética anual de 1 a 3\%. Portanto, a mudança genética anual de P550 dos animais deste rebanho está bastante abaixo do limite inferior do intervalo estabelecido por este autor.

Assim, ao se considerar a discussão sobre os valores genéticos para $\mathrm{P} 550$ e CE, verificou-se que as mudanças genéticas anuais no período avaliado não foram suficientes para se considerar que esta população esteja evoluindo, geneticamente, em termos de aumento no peso ao sobreano e da circunferência escrotal. Dessa forma, maior atenção deve ser dada à seleção dos indivíduos utilizados, sobretudo para a reprodução.

Para os EVs (Figura 1), o comportamento dos valores genéticos diretos foi similar ao verificado para CE, com médias de 0,013 para $\mathrm{C}, 0,010$ para $\mathrm{P}$ e 0,012 para $\mathrm{M}$, para ganho genético anual. Isso representa, respectivamente, $0,39,0,28$ e $0,35 \%$ da média fenotípica, o que foi retratado pela não significância dos valores ( $p<0,214, p<0,342$ e $p<0,483$, respectivamente) e evidencia variabilidade no progresso genético desta população. Para U, este valor foi de 0,017 , o que representa $0,49 \%$ da média fenotípica; porém, apesar de sua amplitude, este valor não foi significativo $(\mathrm{p}<0,159)$, o que indica que a evolução genética para essas características, nesta população, foi nula no período em estudo. Entretanto, como foram constatadas tendências de aumentos discretos nos EVs, como pode ser visualizado pelas retas de regressão dos valores genéticos diretos sobre o ano de nascimento dos animais para C, P, M e U (Figura 1), não houve relação positiva com suas respectivas médias fenotípicas (Figura 2), que apresentaram diminuição constante durante todo o período avaliado.

As tendências fenotípicas para P550 e CE (Figura 2) foram positivas, o que representa aumento de 122,4 gramas por ano, no P550, e de $0,04 \mathrm{~cm}$ por ano na $\mathrm{CE}$. Isso indica que o progresso verificado é decorrente de melhorias nas condições de ambiente, uma vez que
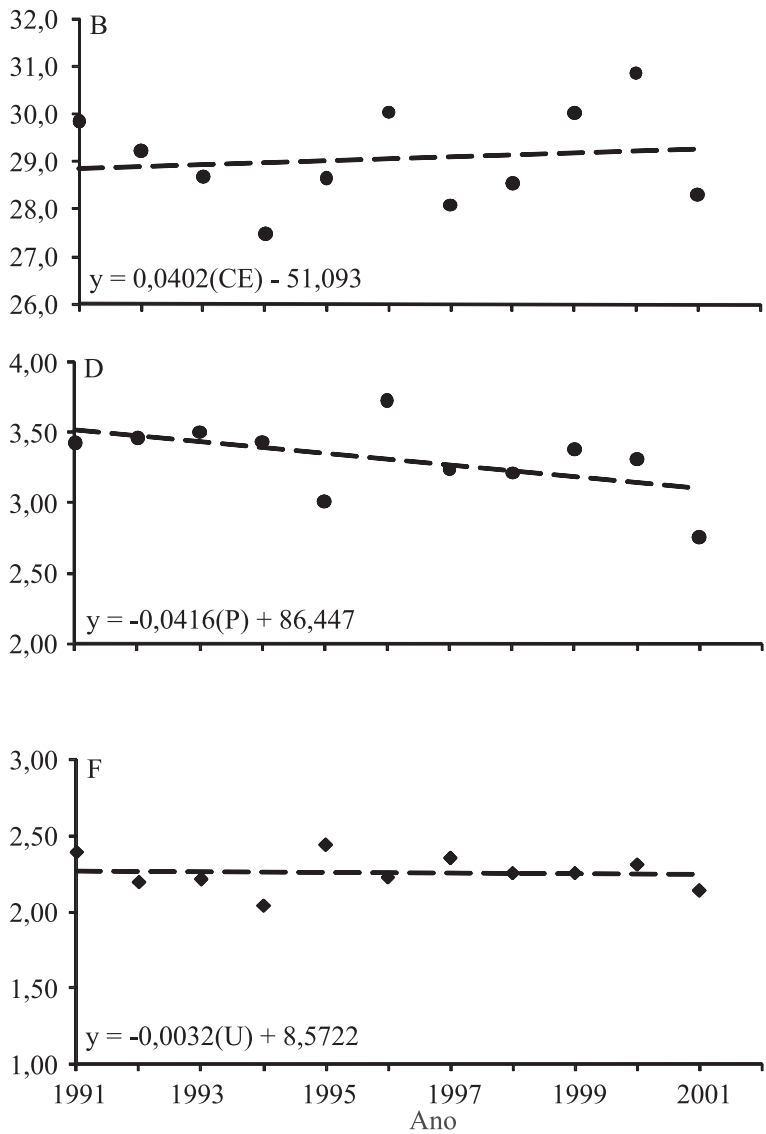

Figura 2. Médias fenotípicas para: A, peso (P550, kg); B, circunferência escrotal (CE, cm); C, conformação (C); D, precocidade (P); E, musculatura (M); e F, umbigo (U), estratificados por unidade de escore, aos 550 dias de idade. 
as mudanças genéticas não foram significativas $(\mathrm{p}<0,14$ para P550 e $\mathrm{p}<0,52$ para $\mathrm{CE}$ ).

A tendência fenotípica foi oposta à genética para todos os EVs, ou seja, houve redução de $-0,0172$, $-0,0416,-0,0557$ e $-0,0032$ pontos por ano (todos com $\mathrm{p}<0,0001$ ), para $\mathrm{C}, \mathrm{P}, \mathrm{M}$ e $\mathrm{U}$, respectivamente, o que pode ser atribuído à inconsistência na classificação desses escores ou à influência ambiental sobre a expressão dessas características. Também é preciso levar em consideração que esses animais foram avaliados durante dez anos, o que corresponde a aproximadamente 2,5 gerações. Assim, recomendamse novas pesquisas com arquivos que representem um período maior de tempo.

Contudo, as tendências fenotípicas para $\mathrm{C}, \mathrm{M}$ e $\mathrm{P}$ podem estar relacionadas às exigências de mercado, como ressaltado por Araújo et al. (2010), pois há demanda por animais que se enquadrem dentro da amplitude observada para estes EVs. No entanto, maior atenção deve ser destinada a P550, CE e U, uma vez que houve diminuição significativa de seus valores no decorrer do período analisado, o que diminuiria a lucratividade do sistema e aumentaria o número de animais com umbigos pendulosos.

Esse comportamento tem sido observado em várias raças, conforme Weber et al. (2009), que obtiveram resultados inferiores para a raça Aberdeen Angus, em que as estimativas para $\mathrm{C}, \mathrm{P}$ e $\mathrm{M}$ foram de 0,0035 , 0,0057 e 0,0026 , respectivamente, em termos de ganho genético anual, tendo representado $0,16,0,10$ e $0,08 \%$ da média fenotípica. Não foram encontradas, na literatura, estimativas de tendências genéticas para $\mathrm{C}, \mathrm{P}, \mathrm{M}$ e $\mathrm{U}$ em bovinos de raças compostas, na pós-desmama, que servissem como base para comparações, o que dificultou a discussão. Porém, para a raça Nelore, Van Melis et al. (2003) relataram valores de tendência genética superiores aos encontrados no presente trabalho para os escores de C $(0,030)$, P $(0,031)$ e $\mathrm{M}(0,030)$. Forni et al. (2007) obtiveram ganhos semelhantes para $\mathrm{C}$, $\mathrm{M}$ e $\mathrm{P}$ à desmama, da ordem de $0,013,0,018$ e 0,022, respectivamente, o que representa $0,42,0,60$ e $0,67 \%$ da média fenotípica dessas características na raça Nelore.

É comum se observar baixos progressos fenotípicos, principalmente quando estimados para rebanhos criados em ambientes distintos, submetidos a diversos sistemas de produção, como é o caso do gado de corte no Brasil (Ferraz Filho et al., 2002). Apesar de os ganhos genéticos obtidos apresentarem valores baixos, o progresso genético deve ser considerado, pois as mudanças genéticas são estáveis, cumulativas e permanentes ao longo dos anos (Weber et al., 2009). Entretanto, embora estáveis, essas mudanças genéticas podem vir a não se expressar, em razão das condições ambientais distintas no decorrer dos anos avaliados. Dessa forma, espera-se que a utilização de índices de seleção para essas características proporcione ganhos genéticos de maior magnitude, uma vez que esses índices consideram as características em conjunto e com valores relativos a sua importância econômica.

O progresso genético para a população estudada foi muito baixo, o que corrobora os resultados obtidos por Lopes et al. (2009). De acordo com estes autores, mesmo quando há progresso anual no desempenho dos animais a partir de melhorias nas condições de meio ambiente, é preciso promover melhoria genética nos próximos anos, caso contrário, o progresso fenotípico atingirá seu limite.

\section{Conclusões}

1. As estimativas dos coeficientes de herdabilidade, para todas as características, indicam considerável variação genética aditiva direta, o que mostra ser possível obter ganho genético por meio da seleção.

2. As correlaçõesgenéticas entre peso ecircunferência escrotal com os escores de conformação, precocidade e musculatura indicam a possibilidade de resposta correlacionada.

3. As tendências genéticas estimadas indicam grande contribuição de fontes de variação não genéticas para todas as características no período estudado, e apontam para necessidade de melhoria nas condições ambientais para que os animais expressem todo seu potencial genético.

\section{Agradecimentos}

À Gensys Consultores Associados e à Natura Genética Sul-Americana, pela disponibilização dos arquivos de dados.

\section{Referências}

ARAÚJO, R.O. de; RORATO, P.R.N.; WEBER, T.; EVERLING, D.M.; LOPES, J.S.; DORNELLES, M. de A. Genetic parameters and phenotypic and genetic trends for weight at weaning and visual 
scores during this phase estimated for Angus-Nellore crossbred young bulls. Revista Brasileira de Zootecnia, v.39, p.2398-2408, 2010.

BOLIGON, A.A.; ALBUQUERQUE, L.G. de; MERCADANTE, M.E.Z.; LÔBO, R.B. Herdabilidades e correlações entre pesos do nascimento à idade adulta em rebanhos da raça Nelore. Revista Brasileira de Zootecnia, v.38, p.2320-2326, 2009.

COSTA, G.Z.; QUEIROZ, S.A.; OLIVEIRA, J.A.; FRIES, L.A. Estimativas de parâmetros genéticos e fenotípicos de escores visuais e de ganho médio de peso do nascimento a desmama de bovinos formadores da raça Brangus. ARS Veterinaria, v.24, p.172-176, 2008.

CYRILLO, J.N. dos S.G.; RAZOOK, A.G.; FIGUEIREDO, L.A. de; BONILHANETO, L.M.; MERCADANTE, M.E.Z.; TONHATI, H. Estimativas de tendências e parâmetros genéticos do peso padronizado aos 378 dias de idade, medidas corporais e perímetro escrotal de machos Nelore de Sertãozinho, SP. Revista Brasileira de Zootecnia, v.30, p.56-65, 2001.

FARIA, C.U.; MAGNABOSCO, C.U.; ALBUQUERQUE, L.G.; REYES, A. de los; BEZERRA, L.A.F.; LÔBO, R.B. Análise bayesiana na estimação de correlações genéticas entre escores visuais e características reprodutivas de bovinos Nelore utilizando modelos linear-limiar. Arquivo Brasileiro de Medicina Veterinária e Zootecnia, v.61, p.949-958, 2009.

FERRAZ FILHO, P.B.; RAMOS, A.A.; SILVA, L.O.C.; SOUZA, J.C.; ALENCAR, M.M. Herdabilidades e correlações genéticas, fenotípicas e ambientais para pesos em diferentes idades de bovinos da raça Tabapuã. Archives of Veterinary Science, v.7, p.75-79, 2002.

FORNI, S.; FEDERICI, J. de F.; ALBUQUERQUE, L.G. de. Tendências genéticas para escores visuais de conformação, precocidade e musculatura à desmama de bovinos Nelore. Revista Brasileira de Zootecnia, v.36, p.572-577, 2007.

GIANOLA, D.; FOULLEY, J.L. Sire evaluation for ordered categorical data with a threshold model. Genetics Selection Evolution, v.15, p.201-224, 1983.

JORGE JÚNIOR, J.; DIAS, L.T.; ALBUQUERQUE, L.G. de. Fatores de correção de escores visuais de conformação, precocidade e musculatura, à desmama, para idade da vaca ao parto, data juliana de nascimento e idade à desmama em bovinos da raça Nelore. Revista Brasileira de Zootecnia, v.33, p.2044-2053, 2004.

KOURY FILHO, W.; ALBUQUERQUE, L.G. de; FORNI, S.; SILVA, J.A. II de V.; YOKOO, M.J.; ALENCAR, M.M. de. Estimativas de parâmetros genéticos para os escores visuais e suas associações com peso corporal em bovinos de corte. Revista Brasileira de Zootecnia, v.39, p.1015-1022, 2010.

KOURY FILHO, W.; JUBILEU, J.S.; ELER, J.P.; FERRAZ, J.B.S.; PEREIRA, E.; CARDOSO, E.P. Parâmetros genéticos para escore de umbigo e características de produção em bovinos da raça Nelore. Arquivo Brasileiro de Medicina Veterinária e Zootecnia, v.55, p.594-598, 2003.

LAUREANO, M.M.M.; BOLIGON, A.A.; COSTA, R.B.; FORNI, S.; SEVERO, J.L.P.; ALBUQUERQUE, L.G. Estimativas de herdabilidade e tendências genéticas para características de crescimento e reprodutivas em bovinos da raça Nelore. Arquivo
Brasileiro de Medicina Veterinária e Zootecnia, v.63, p.143-152, 2011.

LOPES, J.S.; RORATO, P.R.N.; WEBER, T.; DORNELLES, M. de A.; COMIN, J.G.; ARAÚJO, R.O. de. Parâmetros genéticos e tendências genética e fenotípica para características de crescimento em uma população da raça Brangus. Revista Brasileira de Zootecnia, v.38, p.662-669, 2009.

MADUREIRA, A.P.; OLIVEIRA, H.N.; ROSA, G.J.M.; BEZERRA L.F.; MARQUES, L.F.A. Inferência bayesiana na predição de valores genéticos do peso aos 365 dias de bovinos de corte. Archivos de Zootecnia, v.58, p.265-275, 2009.

MISZTAL, I. BLUPF90 family of programs. 2008. Available at: $<$ http://nce.ads.uga.edu/ ignacy/newprograms.html $>$. Accessed on: 6 June 2012.

MUCARI, T.B.; OLIVEIRA, J.A. de. Análise genético-quantitativa de pesos aos 8, 12, 18 e 24 meses de idade em um rebanho da raça Guzerá. Revista Brasileira de Zootecnia, v.32, p.1604-1613, 2003.

OLIVEIRA, L.Z.; OLIVEIRA, C.S.; MONTEIRO, F.M.; LIMA, V.F.M.H. de; LIMA, F.M. de; COSTA, M.Z. Efeito da idade sobre as principais características andrológicas de touros Brangus-Ibagé criados extensivamente no estado do Mato Grosso do Sul - Brasil. Acta Scientiae Veterinariae, v.39, p.1-8, 2011.

QUEIROZ, S.A.; OLIVEIRA, J.A.; COSTA, G.Z.; FRIES, L.A. Estimates of genetic parameters for visual scores and daily weight gain in Brangus animals. Animal, v.5, p.838-843, 2011.

SAS INSTITUTE. SAS/STAT user's guide. Version 9.1.3. Cary: SAS Institute, 2003.

SMITH, B.J. boa: an R package for MCMC output convergence assessment and posterior inference. Journal of Statistical Software, v.21, p.1-37, 2007

SMITH, C. Effects of changes in economic weights on the efficiency of index selection. Journal of Animal Science, v.56, p.1057-1064, 1983.

TORAL, F.L.B.; ALENCAR, M.M. de; FREITAS, A.R. de. Abordagens freqüentista e bayesiana para avaliação genética de bovinos da raça Canchim para características de crescimento. Revista Brasileira de Zootecnia, v.36, p.43-53, 2007.

TSURUTA, S.; MISZTAL, I. THRGIBBS1F90 for estimation of variance component with threshold and linear models. In: WORLD CONGRESS ON GENETICS APPLIED TO LIVESTOCK PRODUCTION, 8., 2006, Belo Horizonte. Proceedings. Belo Horizonte: UFMG, 2006. p.27-31.

VAN KAAM, J.B.C.H.M. Gibanal: analyzing program for Markov Chain Monte Carlo sequences. Version 2.3. Wageningen: Wageningen University, 1997.

VAN MELIS, M.H.; ELER, J.P.; SILVA, J.A. II de V.; FERRAZ, J.B.S. Estimação de parâmetros genéticos em bovinos de corte utilizando os métodos de máxima verossimilhança restrita e R. Revista Brasileira de Zootecnia, v.32, p.1624-1632, 2003.

VIU, M.A.O.; TONHATI, H.; CERÓN-MUNÕZ, M.F.; FRIES, L.A.; TEIXEIRA, R.A. Parâmetros genéticos do peso e escores visuais de prepúcio e umbigo em gado de corte. Ars Veterinaria, v.18, p.179-184, 2002. 
WEBER, T.; RORATO, P.R.N.; LOPES, J.S.; COMIN, J.G.; DORNELLES, M. de A.; ARAÚJO, R.O. de. Parâmetros genéticos e tendências genéticas e fenotípicas para características produtivas e de conformação na fase pré-desmama em uma população da raça Aberdeen Angus. Revista Brasileira de Zootecnia, v.38, p.832-842, 2009.
YOKOO, M.J.I.; ALBUQUERQUE, L.G. de; LÔBO, R.B.; SAINZ, R.D.; CARNEIRO JÚNIOR, J.M.; BEZERRA, L.A.F.; ARAUJO, F.R. da C. Estimativas de parâmetros genéticos para altura do posterior, peso e circunferência escrotal em bovinos da raça Nelore. Revista Brasileira de Zootecnia, v.36, p.1761-1768, 2007.

$\overline{\text { Recebido em } 21 \text { de dezembro de } 2011 \text { e aprovado em } 17 \text { de julho de } 2012}$ 